\title{
SATISFACCIÓN DE LA USUARIA EN CONSULTA EXTERNA DEL SERVICIO DE GINECOLOGÍA EN EL INSTITUTO NACIONAL MATERNO PERINATAL
}

\author{
Jovanna Ochante Rementería ${ }^{1}$, Gilda Hinojosa Flores², Félix Dasio Ayala Peralta ${ }^{3}$, \\ Carlos Perez Aliaga ${ }^{4}$
}

\begin{abstract}
RESUMEN
Objetivos. Determinar el nivel de satisfacción de la usuaria en consulta externa de ginecología. Materiales y métodos. Estudio observacional, descriptivo, retrospectivo y transversal. Encuesta estructurada para 126 pacientes comprendidas de 19 a 65 años de edad atendidas en Consulta Externa de Ginecología en el Instituto Nacional Materno Perinatal. Resultados. El 71\% de las usuarias en consulta externa del servicio de ginecología presentan una satisfacción moderada, el $25,4 \%$ una satisfacción alta y solo un $18,3 \%$ satisfacción baja o insatisfacción. Satisfacción alta tuvieron en ginecología oncológica y mamaria $(80 \%)$, moderada satisfacción el consultorio de ginecología general $(70,3 \%)$. Mayor porcentaje de satisfacción baja o insatisfacción fue en ecografía ginecológica (25\%). El tiempo de espera fue mayor a 30 minutos en el $53,2 \%$ de usuarias y fue en consulta de ginecología oncológica ( $85 \%)$,ecografía ginecológica $(75 \%)$, ginecología general (54\%) e infertilidad (41\%); mientras que $46,8 \%$ esperaron menos de 30 minutos para su atención médica, sobre todo en consulta de climaterio y menopausia (85\%). Hubo suficiente comunicación médico - paciente en el $87,3 \%$ y solo el $12,7 \%$ afirmó que era insuficiente dicha relación. La confidencialidad o privacidad se cumplieron en el $99,2 \%$. En general el $97,6 \%$ de pacientes manifestaron su satisfacción personal con relación a la limpieza de los ambientes de los consultorios externos. Conclusiones. La gran mayoría de usuarias de consulta externa ginecológica presentan nivel moderada a alta satisfacción con suficiente comunicación médico - paciente y cumplimiento de la confidencialidad o privacidad durante la atención médica.
\end{abstract}

Palabras clave: Satisfacción; Usuaria; Paciente ginecológica; Consulta externa (Fuente: DeCS BIREME).

\section{USER SATISFACTION IN OUTPATIENT GYNECOLOGY SERVICE IN THE NATIONAL MATERNAL PERINATAL INSTITUTE}

\begin{abstract}
Objectives. Determine the level of user satisfaction in outpatient gynecology. Materials and methods. Observational, descriptive, retrospective and cross-sectional study. Survey structured for 126 patients ranging from 19-65 years old attended in Outpatient Gynecology at the National Maternal and Perinatal Institute. Results. $71 \%$ of users in outpatient department of gynecology are moderately satisfied, $25.4 \%$ high satisfaction and only $18.3 \%$ low satisfaction or dissatisfaction. Oncológicay had high satisfaction in gynecology breast $(80 \%)$, moderate satisfaction the general gynecology clinic $(70.3 \%)$. Higher percentage of low satisfaction or dissatisfaction was in gynecologic ultrasound (25\%). The waiting time was more than 30 minutes in $53,2 \%$ of users and was in consulting gynecologic oncology $(85 \%)$, gineoclógica ultrasound $(75 \%)$, general gynecology (54\%) and infertility (41\%); while $46,8 \%$ expected less than 30 minutes for their health care, especially in view of menopause and menopause (85\%). There was enough doctor - patient communication in $87.3 \%$ and only $12.7 \%$ said it was insufficient that relationship. Confidentiality or privacy were met in $99.2 \%$. Overall $97,6 \%$ of patients expressed personal satisfaction regarding the cleanliness of the outpatient environments. Conclusions. The vast majority of users of gynecological outpatients with moderate satisfaction levels high enough doctor - patient communication and compliance with confidentiality or privacy during medical care.
\end{abstract}

Key words: Satisfaction; User; Gynecological patient; Outpatient (Source: MeSH NLM).

\section{INTRODUCCIÓN}

La calidad en los servicios de salud está íntimamente ligada con la satisfacción del usuario a los servicios de salud, por ende la satisfacción del usuario es considerado como un indicador de calidad en servicios de salud.
En la actualidad temas como la seguridad del paciente y la calidad de la atención son abordados en el ámbito internacional y nacional, como problema grave de salud pública en todo el mundo. Se calcula que en los países desarrollados hasta uno de cada 10 pacientes sufre algún tipo de daño durante su estancia en el hospital.

Bachiller en Trabajo Social- Universidad Nacional Mayor de San Marcos. Asistente Ejecutiva del Instituto Nacional Materno Perinatal de Lima-Perú

2 Licenciada en Enfermería. Docente de la Facultad de Enfermería - Universidad de Ciencias y Humanidades. Asistente de la Oficina de Gestión de la Calidad del Instituto Nacional Materno Perinatal de Lima-Perú.

3 Médico GínecoObstetra. Magíster en Salud Reproductiva. Profesor de la Facultad de Medicina Humana de la Universidad San Martín de Porres y de Ciencias de la Salud de la Universidad Norbert Wiener. Director de la Oficina de Cooperación Científica Internacional-Instituto Nacional Materno Perinatal de Lima-Perú.

4 Médico Gíneco Obstetra. Director de la Oficina Gestión de la Calidad-Instituto Nacional Materno Perinatal. Lima-Perú.

Recibido: 14-01-15 Aprobado: 12-08-15


Para evaluar este indicador es necesario considerar lo más importante en el proceso de atención sanitaria; como es la gran complejidad del proceso de satisfacción de los usuarios, las características de la organización de salud y la correcta aplicación de la metodología científica al evaluar los indicadores de satisfacción de los usuarios ${ }^{1}$.

El análisis sistemático sobre la percepción de los usuarios con respecto al servicio recibido es fundamental, para evaluar la gestión de una organización en sus diferentes niveles; desde el nivel macro donde se generan las políticas y las normativas, hasta el nivel operativo donde se presta el servicio; de esta forma poder hacer los ajustes necesarios para ofrecer servicios eficientes, competitivos y con una base de respeto por los derechos de los usuarios ${ }^{2}$.

La calidad de la atención de salud es un tema que adquiere cada vez más relevancia debido a la libertad con que opinan los usuarios acerca de los servicios recibidos de los encargados de prestar servicios de salud y de las propias instituciones proveedoras de estos servicios. Esta situación condicionada por los retos organizacionales y la creciente presión social determinada por la toma de conciencia de una sociedad más informada de sus derechos ${ }^{3}$. Asimismo, sirve como indicador para evaluar las intervenciones de los servicios sanitarios pues nos proporciona información sobre la calidad percibida en los aspectos de estructura, procesos y resultados ${ }^{4}$.

La medición de la satisfacción de las usuarias externas en los diferentes servicios de salud, resulta indispensable para la implementación de acciones o proyectos de mejora, que contribuirán con el logro de los objetivos sanitarios con una buena calidad en la atención. De la magnitud de esta diferencia o brecha entre expectativa y percepción va depender mucho la opinión subjetiva favorable o desfavorable de los servicios de salud y de la institución en general.

Conocer la calidad percibida del servicio o la satisfacción experimentada, permitirán efectuar los ajustes necesarios al interior de la organización hospitalaria para que tanto los trabajadores de salud y los directivos armonicen la atención protocolizada durante la prestación del servicio y de las diferentes necesidades de apoyo de quienes participan en la atención de un paciente.

En este sentido, el presente estudio tiene por objetivo identificar la prevalencia de los factores que condicionan la satisfacción de las usuarias, en pacientes atendidas en consulta ambulatoria del Servicio de Ginecología del INMP.

\section{MATERIALES Y MÉTODOS}

Se realizó un estudio observacional, descriptivo y transversal. Se aplicó una encuesta estructurada que consta de 17 ítems para 126 pacientes ginecológicas, cuyas edades son de 19 a 65 años, atendidas en los consultorios externos de ginecología general, climaterio y menopausia, infertilidad, ecografía ginecológica y ginecología oncológica del Instituto Nacional Materno Perinatal durante el periodo de mayo 2015.

Se abordó aspectos sociodemográficos como edad, ocupación, estado civil, grado de instrucción, lugar de procedencia y datos específicos que aborda la calidad de la atención de salud considerando sus tres dimensiones: dimensión humana, dimensión técnicocientífica y dimensión del entorno de la calidad.

Criterios de Inclusión:

Pacientes ginecológicas.

Edad reproductiva (19 a 49 años).

Edad post menopáusica (50 a 65 años).

Adulta Mayor (65 a más)

Criterios de Exclusión:

Pacientes adolecentes.

Gestantes.

Para la graduación del nivel de satisfacción se consideró las siguientes variables:

Satisfacción alta: considera las escalas de siempre y frecuentemente.

Moderada satisfacción: considera la escala algunas veces.

Satisfacción baja o Insatisfecha: considera las escalas rara vez y nunca

El análisis estadístico se realizó en el programa SPSS versión 20.0, posteriormente se elaboraron tablas y gráficos a fin de presentar organizadamente los resultados obtenidos.

\section{RESULTADOS}

Durante el periodo de estudio se registraron 1,260 consultas ginecológicas. Dentro las variables sociodemográficas encontradas fueron: edad de usuarias en promedio $38,4 \pm 9,9$ años, que fluctuó entre los 19 y 65 años; el grupo etario más frecuente fue de 30 a 40 años con un $44,4 \%$, seguido de las que tienen más de 40 años con un $35,7 \%$. El estado civil más frecuente fueron las casadas $(38,1 \%)$, luego las convivientes $(35,7 \%)$, seguido de las solteras $(23 \%)$. El grado de instrucción corresponde a secundaria en $54,8 \%$ y $37,3 \%$ a instrucción superior y solo un $7,9 \%$ a primaria. La ocupación más frecuente fue ama de casa con un $46,8 \%$ y dependientes en un $28,6 \%$ ( tabla 1 ).

Según el tipo de consultorio externo donde las usuarias fueron atendidas son ginecología general el $29,4 \%$, medicina reproductiva $23 \%$, climaterio, ginecología oncológica y ecografía ginecológica en el 15,9\% respectivamente (tabla 2 ). 
Tabla 1. Características sociodemográficas de las usuarias en Consultorio Externo del Servicio de Ginecología en el Instituto Nacional Materno Perinatal-Mayo 2015.

\begin{tabular}{|c|c|c|}
\hline $\begin{array}{c}\text { Características } \\
\text { Sociodemográficas }\end{array}$ & $\mathrm{X} \pm \mathrm{SD}$ & Min-Max \\
\hline Edad (años) & $38,4 \pm 9,9$ & $19-65$ \\
\hline Edad Agrupada & $\mathbf{N}$ & $\%$ \\
\hline$<30$ años & 25 & 19,8 \\
\hline $30-40$ años & 56 & 44,4 \\
\hline >40 años & 45 & 35,7 \\
\hline \multicolumn{3}{|l|}{ Estado Civil } \\
\hline Soltera & 29 & 23 \\
\hline Conviviente & 45 & 35,7 \\
\hline Casada & 48 & 38,1 \\
\hline Otros & 4 & 3,2 \\
\hline \multicolumn{3}{|l|}{ Grado de Instrucción } \\
\hline Primaria & 10 & 7,9 \\
\hline Secundaria & 69 & 54,8 \\
\hline Superior & 47 & 37,3 \\
\hline \multicolumn{3}{|l|}{ Ocupación } \\
\hline Ama de casa & 59 & 46,8 \\
\hline Dependiente & 36 & 28,6 \\
\hline Estudiante & 8 & 6,3 \\
\hline Independiente & 22 & 17,5 \\
\hline No especifica & 1 & 0,8 \\
\hline Total & 126 & 100,0 \\
\hline
\end{tabular}

Tabla 2. Según el tipo de consultorio externo de ginecología en donde la usuaria es atendida en el Instituto Nacional Materno Perinatal-Mayo 2015.

\begin{tabular}{lcc}
\hline \multicolumn{1}{c}{ Tipo de consultorio ginecológico } & N & \% \\
\hline Ginecología general & 37 & 29,4 \\
Medicina reproductiva & 29 & 23,0 \\
Ginecología oncológica & 20 & 15,9 \\
Climaterio y menopausia & 20 & 15,9 \\
Ecografía ginecológica & 20 & 15,9 \\
Total & $\mathbf{1 2 6}$ & $\mathbf{1 0 0 , 0}$ \\
\hline
\end{tabular}

En cuanto a la comunicación del médico hacia la usuaria o sus familiares, el $87,3 \%$ afirmó que el médico si realizaba una comunicación integral con ellas o con sus familiares, mientras que solo el $12,7 \%$ afirmó que no era suficiente dicha comunicación (tabla 3 ).

El tiempo de espera de atención en consultorio externa de ginecología fue mayor a 30 minutos $(53,2 \%)$. Mientras 59 pacientes $(46,8 \%)$ esperaron menos de 30 minutos para su atención (tabla 4).

Tabla 3. Comunicación del médico hacia la usuaria o sus familiares en consultorio externo del servicio de ginecología en el Instituto Nacional Materno PerinatalMayo 2015.

\begin{tabular}{|c|c|c|}
\hline $\begin{array}{c}\text { Comunicación del médico hacia la usuaria } \\
\text { o sus familiares }\end{array}$ & $\mathbf{N}$ & $\%$ \\
\hline $\mathrm{Si}$ & 110 & 87,3 \\
\hline No & 16 & 12,7 \\
\hline Total & 126 & 100,0 \\
\hline
\end{tabular}

Tabla 4. Tiempo de espera para atenderse de las usuarias en consultorio externo del servicio de ginecología en el Instituto Nacional Materno Perinatal-Mayo 2015.

\begin{tabular}{|c|c|c|}
\hline $\begin{array}{l}\text { Tiempo de espera para atenderse } \\
\text { en consulta externa }\end{array}$ & $\mathbf{N}$ & $\%$ \\
\hline$<30$ minutos & 59 & 46,8 \\
\hline$>30$ minutos & 67 & 53,2 \\
\hline Total & 126 & 100,0 \\
\hline
\end{tabular}

Además, según el tipo de consultorio, el tiempo de espera mayor a 30 minutos fue más en consultorio de ginecología oncológica en el $85 \%$, seguida de ecografía ginecológica en $75 \%$; y por el contrario, en climaterio y menopausia, las usuarias afirmaron en su mayoría que el tiempo de espera para ser atendida fue menor a 30 minutos $(85 \%)$ seguida de medicina reproductiva en el $58,6 \%$ (Tabla 5 ).

Tabla 5. Tiempo de espera según el tipo de consultorio ginecológico en el Instituto Nacional Materno PerinatalMayo 2015.

\begin{tabular}{|c|c|c|c|c|c|c|c|c|c|c|}
\hline \multirow{3}{*}{$\begin{array}{c}\text { Tiempo } \\
\text { de } \\
\text { espera }\end{array}$} & \multicolumn{10}{|c|}{ Tipo de Consultorio ginecológico } \\
\hline & \multicolumn{2}{|c|}{ General } & \multicolumn{2}{|c|}{$\begin{array}{l}\text { Ginecología } \\
\text { Oncológica }\end{array}$} & \multicolumn{2}{|c|}{$\begin{array}{c}\text { Medicina } \\
\text { Reproductiva }\end{array}$} & \multicolumn{2}{|c|}{$\begin{array}{l}\text { Climaterio y } \\
\text { menopausia }\end{array}$} & \multicolumn{2}{|c|}{$\begin{array}{c}\text { Ecografía } \\
\text { ginecológica }\end{array}$} \\
\hline & $\mathbf{N}$ & $\%$ & $\mathbf{N}$ & $\%$ & $\mathbf{N}$ & $\%$ & $\mathbf{N}$ & $\%$ & $\mathbf{N}$ & $\%$ \\
\hline $\begin{array}{c}<30 \\
\text { minutos }\end{array}$ & 17 & 45,9 & 3 & 15 & 17 & 58,6 & 17 & 85 & 5 & 25 \\
\hline $\begin{array}{l}>30 \\
\text { minutos }\end{array}$ & 20 & 54,1 & 17 & 85 & 12 & 41,4 & 3 & 15 & 15 & 75 \\
\hline Total & 37 & 100,0 & 20 & 100,0 & 29 & 100,0 & 20 & 100,0 & 20 & 100,0 \\
\hline
\end{tabular}

En relación al nivel de satisfacción de las usuarias de consulta ginecológica se encontró que el $56,3 \%$ de ellas tuvieron una satisfacción moderada seguida del 25,4\% con una satisfacción alta y solo un $18,3 \%$ baja (tabla 6 ).

Tabla 6. Nivel de satisfacción global de las usuarias en consulta externa del Servicio de Ginecología en el Instituto Nacional Materno Perinatal-Mayo 2015.

\begin{tabular}{|c|c|c|}
\hline Nivel de satisfacción & $\mathbf{N}$ & $\%$ \\
\hline Alta & 32 & $25,4 \%$ \\
\hline Moderada & 71 & $56,3 \%$ \\
\hline Baja & 23 & $18,3 \%$ \\
\hline Total & 126 & $100,0 \%$ \\
\hline
\end{tabular}

Como se aprecia en la tabla 7, el nivel de satisfacción según tipo de consultorio ginecológico, encontramos que la satisfacción en las usuarias en ginecología oncológica fue alta en $80 \%$, seguida de climaterio y menopausia en el $25 \%$. Fue moderada la satisfacción en ginecología general en el $70,3 \%$ seguida de ecografía ginecológica en $70 \%$ y climaterio y menopausia en el $60 \%$. Mientras que fue baja satisfacción en consultorio de Ecografía Ginecológia, Medicina reproductiva, Ginecología general, Climaterio y Menopausia, y Ginecología Oncológica en el 25\%, 24,1\%, 16,2\%, 15\% y $10 \%$ respectivamente. 
Tabla 7. Nivel de satisfacción de usuarias según el tipo de Consultorio de Ginecología en el Instituto Nacional Materno Perinatal-Mayo 2015.

\begin{tabular}{|c|c|c|c|c|c|c|c|c|c|c|}
\hline \multirow{3}{*}{$\begin{array}{l}\text { Nivel de } \\
\text { satisfacción }\end{array}$} & \multicolumn{10}{|c|}{ Consultorio ginecológico } \\
\hline & \multicolumn{2}{|c|}{ General } & \multicolumn{2}{|c|}{$\begin{array}{l}\text { Ginecología } \\
\text { Oncológica }\end{array}$} & \multicolumn{2}{|c|}{ Medicina Reproductiva } & \multicolumn{2}{|c|}{$\begin{array}{l}\text { Climaterio y } \\
\text { menopausia }\end{array}$} & \multicolumn{2}{|c|}{$\begin{array}{c}\text { Ecografía } \\
\text { ginecológica }\end{array}$} \\
\hline & $\mathbf{N}$ & $\%$ & $\mathbf{N}$ & $\%$ & $\mathbf{N}$ & $\%$ & $\mathbf{N}$ & $\%$ & $\mathbf{N}$ & $\%$ \\
\hline Baja & 6 & 16,2 & 2 & 10,0 & 7 & 24,1 & 3 & 15 & 5 & 25 \\
\hline Moderada & 26 & 70,3 & 2 & 10,0 & 17 & 58,6 & 12 & 60 & 14 & 70 \\
\hline Alta & 5 & 13,5 & 16 & 80,0 & 5 & 17,2 & 5 & 25 & 1 & 5 \\
\hline Total & 37 & 100,0 & 20 & 100,0 & 29 & 100,0 & 20 & 100,0 & 20 & 100,0 \\
\hline
\end{tabular}

Como se aprecia en la tabla 8 , donde se identifica las preguntas formuladas a las usuarias, los resultados obtenidos de las preguntas del 1 al 6 , hubo mayor porcentaje de usuarias satisfechas que las no satisfechas en todos los casos, aquella pregunta que obtuvo mayor satisfacción fue el ítem $6(99,2 \%)$, sin embargo la que obtuvo mayor porcentaje de insatisfacción fue para la pregunta $2(46,8 \%)$ donde la demora en la atención sobrepasó los 30 minutos de espera.

En relación a la comunicación médico paciente se aprecia que el $87,3 \%$ de pacientes encuestadas manifestaron que se mantuvo suficiente comunicación verbal durante la consulta médica y el $12,7 \%$ no tuvo suficiente comunicación sobre su problema de salud.

Tabla 8. Características del tiempo de espera, comunicación médico-paciente, examen físico y confidencialidad durante la atención recibida. Resultados de la pregunta 1 al 6 de la encuesta realizada a las usuarias en consultorio externo del servicio de ginecología en el Instituto Nacional Materno Perinatal-Mayo 2015.

\begin{tabular}{|c|c|c|}
\hline $\begin{array}{l}\text { P1: ¿Usted fue atendida de manera } \\
\text { inmediata a su llegada al Consultorio } \\
\text { Externo de Ginecología? }\end{array}$ & $\mathbf{N}$ & $\%$ \\
\hline $\mathrm{Si}$ & 89 & 70,6 \\
\hline No & 37 & 29,4 \\
\hline \multicolumn{3}{|c|}{$\begin{array}{l}\text { P2: El tiempo de espera para atenderse en } \\
\text { consulta externa fue más de } 30 \text { minutos }\end{array}$} \\
\hline $\mathrm{Si}$ & 67 & 53,2 \\
\hline No & 59 & 46,8 \\
\hline \multicolumn{3}{|c|}{ P3: ¿La atención en ventanilla de admisión fue rápida? } \\
\hline $\mathrm{Si}$ & 86 & 68,3 \\
\hline No & 40 & 31,7 \\
\hline \multicolumn{3}{|c|}{$\begin{array}{l}\text { P4: ¿El médico que la atendió mantuvo suficiente } \\
\text { comunicación con usted o sus familiares para explicarles } \\
\text { sobre su problema de salud? }\end{array}$} \\
\hline $\mathrm{Si}$ & 110 & 87,3 \\
\hline No & 16 & 12,7 \\
\hline
\end{tabular}

P5: ¿El médico que la atendió realizó un examen físico completo y minucioso por el problema de salud por el cual fue atendida?

$\mathrm{Si} \quad 105 \quad 83,3$

No

21

P6: ¿Durante su atención en Consulta Externa de Ginecología se respetó su privacidad?

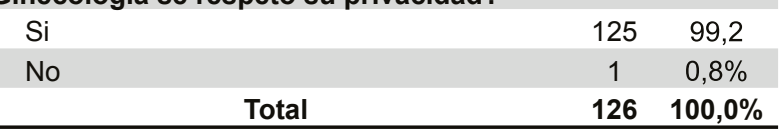

Asimismo, con relación a la confidencialidad o privacidad durante la atención médica en la consulta externa el 99,2\% de pacientes ginecológicas manifestaron que respetaron dicho derecho de la usuaria.

Como se aprecia en la tabla 9, las preguntas del 7 al 12, sucedió lo mismo que lo dicho anteriormente, hubo mayor porcentaje de usuarias satisfechas en todos los ítems que no satisfechas, siendo el ítem 7 y 8 relacionado a la comprensión sobre el tratamiento médico y los análisis y procedimientos a realizarse, arrojó mayor satisfacción ( $97,6 \%$ en ambos casos), y el ítem con mayor porcentaje insatisfacción fue relacionado a la pregunta 11 relacionado a la resolución o mejoría de su problema de salud $(20,6 \%)$.

Asimismo, con relación a la pregunta sobre la limpieza de los ambientes de los consultorios externos, el 97,6\% manifestaron su satisfacción personal.

Tabla 9. Características de la comunicación Médico-Paciente. Resultados de la pregunta 7 al 12de la encuesta realizada a las usuarias en consultorio externo del servicio de ginecología en el Instituto Nacional Materno Perinatal-Mayo 2015.

P7: ¿Usted comprendió la explicación que le brindó el médico sobre el tratamiento que recibió: $\quad \mathrm{N} \quad \%$ tipo de medicamentos, dosis y efectos adversos?

$\begin{array}{ccc}\mathrm{Si} & 123 & 97,6 \\ \mathrm{No} & 3 & 2,4\end{array}$

P8: ¿Ud. Comprendió la explicación que le brindó el médico sobre el análisis y procedimientos que le realizaron?

$\begin{array}{lrl}\mathrm{Si} & 123 & 97,6\end{array}$

No $\quad 3 \quad 2,4$

P9: ¿Ud. Comprendió la explicación que le brindó el médico sobre el diagnóstico de su enfermedad?

$\begin{array}{lcc}\mathrm{Si} & 117 & 92,9\end{array}$

No

$9 \quad 7,1$

P10: ¿El médico que la atendió le brindó el tiempo necesario para contestar sus dudas o preguntas sobre su enfermedad?

$\begin{array}{lll}\mathrm{Si} & 108 & 85,7\end{array}$

No $\quad 18 \quad 14,3$

P11: ¿El problema de salud por el cual usted fue atendida fue resuelto o mejorado?

$\begin{array}{lcc}\mathrm{Si} & 100 & 79,4 \\ \mathrm{No} & 26 & 20,6\end{array}$

P12: ¿Los ambientes de Consultorios Externos estuvieron limpios y cómodos?

\begin{tabular}{|c|c|c|}
\hline $\mathrm{Si}$ & 123 & 97,6 \\
\hline No & 3 & 2,4 \\
\hline
\end{tabular}


Como se aprecia en la tabla 10 , sobre las características del entorno de la consulta externa, en los resultados de las preguntas del 13 al 18 , se puede notar que hubo mayor porcentaje de usuarias satisfechas en todos los ítems, siendo el que mayor porcentaje tiene la pregunta 18 $(99,2 \%)$, y el ítem 14 relacionado a la presencia de avisos o letreros que sirvan de guía para encontrar el consultorio arrojó con mayor porcentaje de usuarias insatisfechas $(46,8 \%)$.

Tabla 10. Características del entorno de la Consulta externa. Resultados de la pregunta 13 al 18de la encuesta realizada a las usuarias en consultorio externo del servicio de ginecología en el Instituto Nacional Materno PerinatalMayo 2015.

\begin{tabular}{|c|c|c|}
\hline $\begin{array}{l}\text { P13: ¿El consultorio médico contó con } \\
\text { equipos disponibles y materiales necesarios } \\
\text { para su atención? }\end{array}$ & $\mathbf{N}$ & $\%$ \\
\hline $\mathrm{Si}$ & 89 & 70,6 \\
\hline No & 37 & 29,4 \\
\hline \multicolumn{3}{|c|}{$\begin{array}{l}\text { P14: ¿Los carteles, letreros y flechas del servicio } \\
\text { ginecología de consultorios externos le parecen } \\
\text { adecuados? }\end{array}$} \\
\hline $\mathrm{Si}$ & 67 & 53,2 \\
\hline No & 59 & 46,8 \\
\hline \multicolumn{3}{|l|}{ P15: ¿La atención en laboratorio fue rápida? } \\
\hline $\mathrm{Si}$ & 86 & 68,3 \\
\hline No & 40 & 31,7 \\
\hline \multicolumn{3}{|l|}{$\begin{array}{l}\text { P16: ¿La atención para tomarse exámenes } \\
\text { radiológicos fue rápida? }\end{array}$} \\
\hline $\mathrm{Si}$ & 110 & 87,3 \\
\hline No & 16 & 12,7 \\
\hline \multicolumn{3}{|c|}{$\begin{array}{l}\text { P17: ¿La farmacia de consultorios externos contó con los } \\
\text { medicamentos que recetó el médico? }\end{array}$} \\
\hline $\mathrm{Si}$ & 105 & 83,3 \\
\hline No & 21 & 16,7 \\
\hline \multicolumn{3}{|c|}{ P18: ¿Está satisfecha con la atención médica recibida? } \\
\hline $\mathrm{Si}$ & 125 & 99,2 \\
\hline No & 1 & 0,8 \\
\hline Total & 126 & $100,0 \%$ \\
\hline
\end{tabular}

Finalmente, destacar que la pregunta 18 relacionada a la satisfacción durante la atención médica recibida, la mayoría absoluta $99,2 \%$ reportan encontrarse satisfechas de la atención de salud recibida en consulta externa de ginecología en el Instituto Nacional Materno Perinatal.

\section{DISCUSION}

El presente estudio destaca la percepción de las usuarias femeninas atendidas en los diferentes consultorios de ginecología para encontrar las expectativas relacionado a la satisfacción del cliente donde influyen múltiples variables.

La satisfacción del usuario implica una experiencia racional o cognoscitiva, derivada de la comparación entre las expectativas y el comportamiento del producto o servicio, está subordinada a numerosos factores como las expectativas, valores morales, culturales, necesidades personales y la propia organización sanitaria. Estos elementos condicionan que la satisfacción sea diferente para distintas personas y para la misma persona en diferentes circunstancias.

Según la literatura Thompson y Col ${ }^{5}$ la satisfacción del usuario depende no solo de la calidad de los servicios sino también de sus expectativas. El usuario está satisfecho cuando los servicios cubren o exceden sus expectativas.

En nuestra casuística el 99,2 \% de usuarias en consulta externa en las diferentes áreas de ginecología reportan la satisfacción personal recibida durante la atención médica. Pero, relacionado al global de otras variables se encontró que el nivel de satisfacción de las usuarias de consulta ginecológica fue una satisfacción moderada en 56,3\% seguida del $25,4 \%$ con una satisfacción alta y solo un $18,3 \%$ baja o insatisfacción. Asimismo, para cada consultorio ginecológico, resultó que la satisfacción en las usuarias fue alta en ginecología oncológica, seguida de climaterio y menopausia. Fue moderada satisfacción en ginecología general seguida de ecografía ginecológica y climaterio y menopausia.

Estos datos concuerdan con lo reportado por Huiza ${ }^{6}$, quién investigó la satisfacción del usuario externo sobre la calidad de atención de salud en consulta externa en el Hospital de la Base Naval del Callao, que involucró todas las especialidades incluidas ginecología y obstetricia que concluyen que el usuario externo estuvo satisfecho con la calidad de atención de salud de la consulta externa en el $76,15 \%($ IC $70,84-81,47 \%)$. La satisfacción estuvo influenciada positivamente por los ítems relacionados con la limpieza y orden del consultorio, y por la limpieza de la sala de espera; y, negativamente por la provisión de Farmacia de todos los medicamentos recetados por el médico y el tiempo de espera.

Diferentes reportes, como Cordova y $\operatorname{col}^{7}$ quien realizó una investigación en 120 usuarios para determinar el grado de satisfacción del usuario externo en el área de emergencia del Hospital Grau en medicina, cirugía y traumatología, en relación con la motivación del personal de salud, a través de encuestas del modelo Servqual modificado por Elías y Alvarez, cuyos resultados del nivel de satisfacción fluctúa entre un máximo de $64,2 \%$ de aseguramiento o cortesía, y un mínimo de $54,9 \%$ en empatía o atención individualizada del usuario, con cifras intermedias en otros ítems como fiabilidad nivel de efectividad (en 63,3\%), tangibilidad o aspecto de las personas e instalaciones físicas $(56,7 \%)$, sensibilidad o tiempo de espera (55\%).

Asimismo, según reporta Tataje $\mathrm{OR}^{8}$ en un estudio para determinar el nivel de satisfacción de la puérpera sobre la atención que brinda la enfermera en el Hospital nacional Docente Madre Niño San Bartolomé, concluye que la mayoría $25(50 \%)$ refiere satisfacción alta, 15 (30\%) nivel medio, $10(20 \%)$ nivel de satisfacción bajo, evidenciado esto principalmente por la relación de confianza apoyo 
emocional, conocimientos que brinda la enfermera. Cabe señalar que en la dimensión biológica, emocional y dimensión espiritual también se obtuvo un porcentaje elevado.

Por otro lado, según reporte de Ortiz y col ${ }^{9}$ en 15 hospitales de México sobre satisfacción de los usuarios muestran que la satisfacción de la atención depende de una serie de circunstancias de orden cultural, social y económico y que la percepción de insatisfacción estuvo relacionada con escasa información sobre el padecimiento actual, con omisiones en las acciones de exploración y diagnóstico, con inadecuadas relaciones interpersonales, es especial de los médicos, y el complicado trámite para obtener la consulta.

El tiempo de espera es un indicador importante en la calidad de un servicio y estos hallazgos influyen positivamente o negativamente en la valoración de la satisfacción de los usuarios en los servicios de salud.

En nuestra investigación, relacionada al tiempo de espera en consulta ginecológica fue mayor a 30 minutos en el $53,2 \%$ y el $46,8 \%$ de usuarias tuvieron menos de 30 minutos de espera; además, según el tipo de consultorio, el tiempo de espera mayor a 30 minutos fue más en consultorio de ginecología oncológica, seguida de ecografía ginecológica; por el contrario, el tiempo de espera fue menor a 30 minutos en climaterio y menopausia seguida de medicina reproductiva. Estos datos concuerdan con lo reportado por Chávez de $\mathrm{Paz}^{10}$ sobre nivel de satisfacción del usuario en los consultorios externos de dermatología del Hospital Nacional Dos de Mayo, que concluye que ante un menor tiempo de espera aumenta la percepción de satisfacción por parte de los pacientes, además la atención médica requiere un tiempo promedio para que pueda ser de calidad, una consulta muy corta o muy larga puede generar pérdidas a los servicios de salud; de este modo, los últimos pacientes se perjudican con un tiempo de espera mayor lo cual es susceptible de ser modificado con una mejor organización.

Se puede concluir que la gran mayoría de usuarias de consulta externa ginecológica presentan nivel moderada a alta satisfacción con suficiente comunicación médico
- paciente y cumplimiento de la confidencialidad o privacidad durante la atención médica recibida en el Instituto Nacional Materno Perinatal.

\section{REFERENCIAS BIBLIOGRÁFICAS}

1. Zas B. La satisfacción como indicador de excelencia en la calidad de los servicios de Salud. Disponible en: www. psicologiacientifica.com/.../psicologiapdf-80-la-satisfaccioncomo-indicador-de-excelencia-en-la-calidad-de-losservicios-de-s.pdf.2002. Enero 2010.

2. Vela CG. Evaluación de la calidad del Servicio de Emergencia de Adultos del Hospital Nacional Edgardo Rebaliati Martins EsSALUD, desde el punto de vista de los usuarios, año 2005. (Tesis para obtener el grado de Doctor). Lima, Perú: Universidad Nacional Mayor de San Marcos, 2010. 212p.

3. Ortiz RM, Muñoz S, Lechuga D, Torres E. Consulta externa en instituciones de salud de Hidalgo, México, según opinión de los usuarios. Revista Panamericana de Salud Pública 2003; 13 (4).

4. Thompson A, Sunol R. Expectations as determinants of patient satisfaction: Concepts, theory and evidence. International Journal for Quality in Health Care 1995; 7(2): 127-141 1995.

5. Cantú H. Desarrollo de una cultura de calidad. Mexico. En: McGraw Hill Interamericana.1997: 166pp.

6. Huiza GA. Satisfacción del usuario externo sobre la calidad de atención de salud en el Hospital de la Base Naval. Callao. Octubre - Diciembre 2003 (Magister en Enfermería mención Gestión en Enfermería). Lima, Perú. Universidad Nacional Mayor de San Marcos, 2006. 87 pp.

7. Córdova VH. Satisfacción del Usuario Externo en el Área de Emergencia del Hospital Graú, en Relación con la Motivación del Personal de Salud (Magistar en Gerencia de Servicios de Salud); Lima, Perú. Universidad Nacional Mayor de San Marcos, 2007; 12pp.

8. Tataje OR. Nivel de satisfacción de la puérpera sobre la atención que brinda la enfermera en el Hospital nacional Docente Madre Niño San Bartolomé. Noviembre 2003. (Licenciada en Enfermería). Lima, Perú. Universidad Nacional Mayor de San Marcos, 2004. 87 pp.

9. Ortiz RM, Muñoz S, Torres E. Satisfacción de los Usuarios de 15 Hospitales de Hidalgo, México. Rev Esp Salud Pública $2004 ; 78$ (4) :1-9.

10. Chávez de Paz P, Ramos W, Galarza C. Nivel de satisfacción del usuario en los consultorios externos de dermatología del Hospital Nacional Dos de Mayo. Dermatol Perú 2009;19(1): 22-31.
ORCID iDs

Félix Ayala Peralta 